\title{
Free-Text Search versus Complex Web Forms
}

\author{
Kien Tjin-Kam-Jet, Dolf Trieschnigg, and Djoerd Hiemstra \\ University of Twente, Enschede, The Netherlands \\ \{tjinkamj,trieschn, hiemstra\}@cs. utwente.nl
}

\begin{abstract}
We investigated the use of free-text queries as an alternative means for searching 'behind' web forms. We conducted a user study where we evaluated our prototype free-text interface in a travel planner scenario. Our results show that users prefer this free-text interface over the original web form and that they are about $9 \%$ faster on average at completing their search tasks.
\end{abstract}

\section{Introduction}

The internet contains a large amount of information that is only accessible through complex web forms. Journey planners, real estate websites, online auction and shopping websites, and other websites commonly require the user to fill out a form consisting of a number of fields in a graphical interface. The user should first interpret the form and then translate his information need to the appropriate fields. Filling out these forms can be slow because they require mixed interaction with both the mouse and keyboard. A natural language interface (NLI) allows the user to enter his information need in a single textual statement. Rather than navigating between and entering information in the components of the web form, the user can focus on formulating his information need in an intuitive way. NLIs require or assume syntactically well-formed sentences as input, in essence restricting the range of textual input. However, describing all possible natural language statements and dealing with query ambiguity can be a time-consuming process [1-4]. Therefore, we introduce a free-text interface (FTI) which allows the user to freely input text without any restrictions. This work is a stepping stone for further investigation of a single textual interface to access the deep web [5]. Ideally, we wish to use these techniques to build a distributed search system which can search multiple resources, including information behind complex web forms, simultaneously. Our contribution is that we demonstrate that users can search faster with an FTI than with a complex web form, and that they prefer the FTI over the complex web form.

\section{Experiment and Results}

We developed a prototype FTI, consisting of a single search box (see Fig. 2), and compared it to an existing travel-planner web form (see Fig. 1). Six information items can be specified in the form: the departure and arrival locations, an optional via location, the time, the date, and a flag indicating whether the date and time are for arrival or departure. In our experiment we try to answer the following questions: 


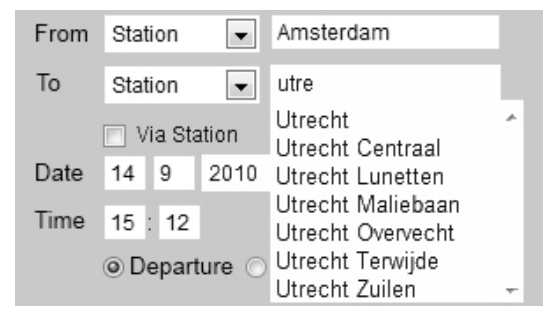

Fig. 1. A complex web form, based on the Dutch Railways site.

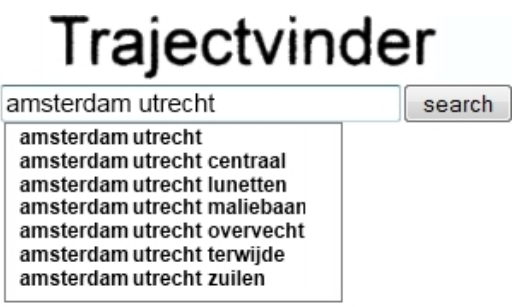

Fig. 2. Trajectvinder ('Route Finder'): an FTI, tailored to the complex web form.

i) do people prefer to use an FTI over a complex web form? ii) is searching by means of an FTI faster than searching by means of a complex web form? iii) is there much variation in the query formulations? $i v$ ) are people consistent in their query formulations? $v$ ) what are the positive and negative aspects of the FTI? and vi) why is the FTI better, or worse, than the complex web form?

\subsection{Experimental Setup}

Experimental procedure. The experiment consisted of an offline part, an online part, and a questionnaire. During the offline part, the subjects first provided background information (e.g. age, study). Then, they wrote down their 'most recent travel question' if they could remember it. Next, an information need was shown as a route on a map, along with a desired date and time. The subjects were asked to fill out the complex web form on paper based on this information need. Likewise, but based on a different information need, they filled out the FTI on paper. Finally, the subjects were shown a filled out complex web form, and they reformulated that into a question suitable for the FTI. We aimed to collect query formulations with as little bias to the question as possible. That is why we asked the subjects to formulate a query both from memory, and based on graphical instead of textual descriptions of the information need. During the online part, the subjects first familiarized themselves with the complex interface of the existing travel planner site. Then, they searched for 5 specific train routes and wrote down the departure and arrival times. We recorded the total time to find all routes. Each route was described textually, with a different order of the information items (i.e. the date, time, and locations), and with different wordings (e.g. ten past one, or 13:10). Next, the subjects familiarized themselves with the FTI. After that, they searched for 5 specific routes and wrote down the departure and arrival times, and we recorded the total search time. All questions in the questionnaire, except for the open questions and explanatory questions, were answered on a five-point Likert scale. The subjects indicated whether they thought the FTI was easy to use, if they could find results faster using the FTI, and whether the results of the FTI were correct. They indicated whether or not the FTI was nicer and better, and explained why they thought so. There were two open questions, asking the subjects to indicate the most negative and the most positive aspects of the system. Finally, they indicated which system they preferred.

Analysis. We tested whether the task completion times of the FTI differed significantly $(p<0.05)$ from those of the complex web form, using the Paired 
Samples T-Test. We also tested whether the five-point Likert scale values differed significantly from neutral (i.e. the number ' 3 '), also using the T-Test. Further, we evaluated the query formulation consistency by looking at the order of the information items. Each item was first replaced by a symbol as follows. We replaced the 'from' (location) with A, 'to' with B, 'via' with $\mathrm{V}$, the 'date' with $\mathrm{D}$, and the 'time' with T. For example, the input "from Amsterdam via Haarlem to The Hague, tomorrow at 10am." was represented as AVBDT. We then measured the correlation between the subject's query formulation and the task description using Kendall's $\tau$. Lastly, for each subject, we measured the average Kendall's $\tau$ over the combinations of the subject's formulations.

\subsection{Results}

The subjects. A total of 17 subjects ( 11 male, 6 female) participated in the study. The age distribution ranged from 21 to 66 (median: 27, mean: 32); most subjects were between the age of 21 and 33 . The background of the subjects ranged from (under)gradate students in various studies to people working in healthcare, consultancy, and IT-software development. Participation (including the questionnaire) took around 30 minutes on average for each subject.

The questionnaire. Comparing the free-text interface (FTI) against the complex web form, the subjects indicated on a five-point Likert scale whether the FTI was: faster (2.4), nicer (1.8), better $(\mathbf{2 . 5})$, and preferred $(\mathbf{2 . 0})$. The numbers in parentheses are the average scores, where ' 1 ' indicates full agreement, and ' 5 ' denotes the opposite. All results differed significantly $(p<0.05)$ from neutral, except for the third aspect. On average, the subjects felt that they could search a little faster using the FTI than using the complex web form. This was supported by the times measured for the web form and the FTI, with $\mathbf{7 . 3}$ and 6.7 minutes on average, respectively. The subjects were significantly $(p=0.032)$ faster, by about $9 \%$, when using the FTI instead of the complex form.

Pros and cons. The subjects listed the most negative and most positive aspects of the FTI. The following negative aspects were mentioned: $24 \%$ of the subjects indicated that there was no example or short manual (forcing the subjects to 'just type in something, and it worked'); $18 \%$ indicated that the interface was too simple, e.g. it lacked pictures; and $12 \%$ disliked that they had to 'clickthrough' to obtain the same results as with the complex web form. The following positive aspects were mentioned: $41 \%$ of the subjects liked how the system 'understood' dates like tomorrow and Tuesday, and written time like 'ten past nine'; $41 \%$ liked that you only had to type (without clicking on menus); $35 \%$ mentioned the query-suggestions as a useful feature; and $18 \%$ appreciated the fact that the input order of information items (e.g. time, date, places) did not matter.

Consistency. When considering only the order of the information items ${ }^{1}$ in a query, there were 17 different query formulations. The three most frequent online query formulations were: ABDT $41 \%$, ABVDT $15 \%$, and, tied at third place with $6 \%$, were ABTD, DTABV, and TABVD.

\footnotetext{
${ }^{1}$ i.e. the 'date' (D), 'time' (T), and the 'from' (A), 'to' (B), and 'via' (V) locations.
} 
The mean Kendall's $\tau$ between the online task descriptions and the query formulations was $\mathbf{0 . 4 2}$. The task with the highest average $\tau(0.96)$ was sequenced ABDT, the other tasks were BADT (0.67), TABVD (0.39), DTABV (0.09), and TBAD (-0.02). Two subjects always followed the same information order of the descriptions and had an average $\tau$ of 1.0 (though they used different wordings). Three subjects had an average $\tau$ between 0.6 and 1.0, and the rest of the seventeen subjects had an average less than or equal to 0.3 .

The mean Kendall's $\tau$ for the (within subjects) online query formulations was 0.64. Six subjects always formulated their questions in the same order and had an average $\tau$ of 1 ; six subjects averaged between 0.7 and 0.9 ; and, five subjects had an average $\tau$ less than 0.2 .

Overall, the subjects were highly consistent in their query formulations individually; however, there was considerable query variation between subjects. Further, the task descriptions had little effect on the subjects' query formulations; the moderate correlation $(0.42)$ is most probably an artifact caused by subjects consistently formulating their queries as ABDT. This explains the high correlations between the query formulations and the two tasks ABDT and BADT.

\section{Conclusion}

We conducted a user study to compare a free-text interface (FTI) with a complex web form in a travel planner scenario. Our results showed that the subjects could search $9 \%$ faster when using the FTI instead of the complex form, and that this finding is significant. Furthermore, they preferred the FTI over the original web form. The results also showed that the subjects were highly consistent in their individual query formulations, and that there was considerable query variation between subjects, even in such a relatively simple scenario.

Acknowledgments. This research was supported by the Netherlands Organization for Scientific Research, NWO, grants 639.022.809 and 612.066.513.

\section{References}

1. Sun, J., Bai, X., Li, Z., Che, H., Liu, H.: Towards a wrapper-driven ontology-based framework for knowledge extraction. In: KSEM'07, Berlin, Heidelberg, SpringerVerlag (2007) 230-242

2. Kaufmann, E., Bernstein, A.: Evaluating the usability of natural language query languages and interfaces to semantic web knowledge bases. Web Semantics: Science, Services and Agents on the World Wide Web (2010)

3. Papakonstantinou, Y., Gupta, A., Garcia-Molina, H., Ullman, J.D.: A query translation scheme for rapid implementation of wrappers. In: DOOD'95, London, UK, Springer-Verlag (1995) 161-186

4. Appelt, D.E., Onyshkevych, B.: The common pattern specification language. In: Proceedings of a workshop on held at Baltimore, Maryland, Morristown, NJ, USA, Association for Computational Linguistics (1996) 23-30

5. Madhavan, J., Ko, D., Kot, L., Ganapathy, V., Rasmussen, A., Halevy, A.: Google's deep web crawl. Proc. VLDB Endow. 1(2) (2008) 1241-1252 